OPEN ACCESS

Edited by:

Enrico Baruffini,

University of Parma, Italy

Reviewed by:

Vincent El Ghouzzi

Institut National de la Santé et de la

Recherche Médicale

(INSERM), France

Muhammad Umair,

Quaid-i-Azam University, Pakistan

Mustafa Ozen,

Baylor College of Medicine,

United States

${ }^{*}$ Correspondence:

Naveed Wasif

naveedwasif@gmail.com

Specialty section

This article was submitted to

Genetic Disorders

a section of the journal

Frontiers in Pediatrics

Received: 28 February 2020

Accepted: 05 June 2020

Published: 16 July 2020

Citation:

Gaboon NEA, Parveen A, Ahmad KA

Shuaib T, Al-Aama JY, Abde/wehab L,

Arif $A$ and Wasif N (2020) A Novel

Homozygous Frameshift Variant in

DYM Causing

Dyggve-Melchior-Clausen Syndrome

in Pakistani Patients.

Front. Pediatr. 8:383.

doi: 10.3389/fped.2020.00383

\section{A Novel Homozygous Frameshift Variant in DYM Causing Dyggve-Melchior-Clausen Syndrome in Pakistani Patients}

\begin{abstract}
Nagwa E. A. Gaboon ${ }^{1}$, Asia Parveen ${ }^{2,3}$, Khaled A. Ahmad ${ }^{4}$, Taghreed Shuaib ${ }^{5}$, Jumana Y. Al-Aama ${ }^{6,7}$, Lereen Abdelwehab ${ }^{8}$, Amina Arif ${ }^{3}$ and Naveed Wasif ${ }^{2,9,10 *}$

${ }^{1}$ Faculty of Medicine, Medical Genetics Center, Ain Shams University, Cairo, Egypt, ${ }^{2}$ Center for Research in Molecular Medicine (CRiMM), Institute of Molecular Biology and Biotechnology (IMBB), The University of Lahore, Lahore, Pakistan ${ }^{3}$ Faculty of Life Sciences, University of Central Punjab (UCP), Lahore, Pakistan, ${ }^{4}$ Department of Radiology, Faculty of Medicine, Ain Shams University, Cairo, Egypt, ${ }^{5}$ Pediatric Department, King Abdulaziz University, Jeddah, Saudi Arabia, ${ }^{6}$ Department of Genetic Medicine, Faculty of Medicine, King Abdulaziz University, Jeddah, Saudi Arabia, ${ }^{7}$ Princess Al-Jawhara Albrahim Center of Excellence in Research of Hereditary Disorders, King Abdulaziz University, Jeddah, Saudi Arabia, ${ }^{8}$ Faculty of Medicine, Ain Shams University, Cairo, Egypt, ${ }^{9}$ Institute of Human Genetics, University of Ulm and University of Ulm Medical Center, Ulm, Germany, ${ }^{10}$ Institute of Human Genetics, University Hospital Schleswig-Holstein, Kiel, Germany
\end{abstract}

Background: Dyggve-Melchior-Clausen syndrome (DMC) is a skeletal dysplasia with associated defects of brain development and intelligence. The truncating pathogenic variants in DYM are the most frequent cause of DMC. Smith-McCort (SMC), another skeletal dysplasia, is also caused by non-synonymous DYM variants.

Methods and Results: In the current study, we examined a Pakistani consanguineous family with three affected members. Clinical features like spondyloepimetaphyseal dysplasia, indicative of characteristic skeletal abnormalities, and intellectual disability were observed. Our male patients had microcephaly and coarse facial features while the female patient did not represent microcephaly or abnormal facies, which are significant features of DMC patients. Sanger sequencing identified a novel homozygous frameshift insertion (c.95_96insT, p.W33Lfs*14) in DYM, which likely leads to nonsense-mediated decay (NMD).

Conclusion: The novel frameshift change verifies the fact that pathogenic variants in DYM are the most frequent cause of DMC.

Keywords: Dyggve-Melchior-Clausen syndrome, Smith-McCort dysplasia, consanguineous, Sanger sequencing, DYM

\section{BACKGROUND}

Dyggve-Melchior-Clausen syndrome (DMC, MIM\#223800) is a rare autosomal recessive skeletal dysplasia with characteristic symptoms of spondyloepimetaphyseal dysplasia (SEMD), mild to severe intellectual disability, microcephaly, coarse facial features, short stature, other skeletal abnormalities like the double-humped appearance of vertebrae and lacy iliac crests (1-5). The absence of other clinical features like corneal opacity and excretion of keratan sulfate in urine and presence of specific radiological findings differentiate it from Morquio's disease (MPS type IV, MIM 253010) (6). 
DMC is caused by an alteration or complete absence of DYMECLIN (DYM) protein expression. The in situ hybridization studies on various embryonic stages of human development have demonstrated that the DYM expresses in human embryonic and fetal tissues during the whole human growth process (7). DYM may have a critical role in the formation and function of the Golgi apparatus and in monitoring the movements of several associated proteins and vesicles across the organelle (8). Dymeclin deficient mice $\left(\mathrm{Dym}^{-/-}\right)$have been reported to develop progressive skeletal deformities, suggesting skeletal phenotypes of DMC and SMC in humans (8). The particular expression of DYM has been noted in the human brain, chondrocytes, osteoblasts, and skin fibroblasts (9).

SMC is a clinical variant of DMC, sharing many skeletal phenotypes except for microcephaly and intellectual disability $(9,10)$. The missense variants in DYM reported in SMC (SMC1, MIM\#607326) patients, may not affect the localization of DYM protein compared to the truncating pathogenic variants causing DMC (9). A complete absence of DYM protein in DMC patients could be suggestive of brain defects $(7,8)$. Mice knockout $\left(\mathrm{Dym}^{-/-}\right)$studied by Dupuis et al. (11), revealed the depletion of Dym protein, leading to defects in brain development and postnatal-onset microcephaly. Recently, a novel gene Ras-Related Protein RAB-33B (RAB33B, MIM\#605950) has also been reported to cause SMC2 (MIM\# 615222) (12).

We have performed a clinical and mutational diagnosis of a consanguineous Pakistani family. Clinical surveys were suggestive of DMC, and the pathogenic variant search revealed a novel homozygous frameshift insertion (c.95_96insT, p.W33Lfs*14) in DYM.

\section{MATERIALS AND METHODS}

\section{Enrollment of Subjects, Extraction of Blood and DNA}

The study included three related Pakistani patients (IV7, IV-10, IV-13) (Figure 1). The patients were clinically evaluated at the Department of Genetic Medicine, King Abdulaziz University (KAU) Hospital, Jeddah, for a genetic consultation over skeletal deformities and intellectual disability. Written informed consent was obtained from the parents of the affected members. A pedigree construction followed detailed family history. A physical examination with particular emphasis on orthopedic findings, anthropometric measurements was done. The skeletal surveys and relevant laboratory investigations were also performed.

Peripheral blood sampling was followed by DNA extraction using the QIAamp DNA Blood Mini kit (Cat No. /ID: 51106, QIAGEN) in ten family members, including three affected and an unaffected member (IV-4, IV-7, IV-10, IV-13) and six unaffected parents (III-2, III-4, III-5, III-7, III-9, III-10). This study was approved, under a reference number 24/14, by the medical ethics and research committee of King Abdulaziz University, Jeddah, Saudi Arabia.

\section{DNA Sequencing}

The reference sequence of DYM (NM_017653.6, NG_009239.2, NP_060123.3) was obtained from the University of California Santa Cruz (UCSC) genome database browser (http://genome. ucsc.edu/cgi-bin/hgGateway). The primer pairs for all 16 coding exons of DYM were designed using AmplifX v1.5.4 software (http://crn2m.univ-mrs.fr/pub/amplifx). The first coding exon was amplified by the following pair of primers (DYM-F: $5^{\prime}$-ggcctagccccatatttcttg-3', melting temperature $\left.61.9^{\circ} \mathrm{C}\right) \quad$ (DYM-R: $5^{\prime}$-aggtggctactgagttgggtaa-3, melting temperature $60.1^{\circ} \mathrm{C}$ ). Another forward primer (DYM-F: $5^{\prime}-$ ggtagacaaagcatgatgacttttgaac- $3^{\prime}$, melting temperature $64.2^{\circ} \mathrm{C}$ ) was used for Sanger sequencing to perform the segregation analysis. PCR amplified the regions of interest, and the cleaning of PCR products was done by Exo-Sap protocol (https://www. thermofisher.com). The BigDye chemistry v3 was used to prepare the sequencing reaction, and DNA sequencing was performed on the ABI3730 genetic analyzer. The alignment of sequencing data against the reference genome was performed by a sequence alignment tool, BioEdit version 6.0.7 (http://www.mbio.ncsu. edu/BioEdit/bioedit.html).

The minor allele frequency (MAF) of the pathogenic variant was verified from the genome aggregation database (gnomAD, http://gnomad-old.broadinstitute.org/). A panel of 96 healthy control samples from the Pakistani population were also Sanger sequenced to exclude the possibility of a common polymorphism. The possible effect of the variant over protein stability was predicted by two online algorithms MutationTaster (http://www. mutationtaster.org/) and PROVEAN (http://provean.jcvi.org/ index.php).

\section{RESULTS}

\section{Clinical Findings Case-I (IV-7)}

The index patient (IV-7) was a 12-years and 7 months old girl, at the time of her visit to the Department of Genetic Medicine, King Abdulaziz University (KAU) Hospital, Jeddah. She is the eighth offspring of a double first cousin Pakistani couple (41years-old mother (III-4) and a 53-years-old father (III-5) at the time of conception). She was born full-term pregnancy with spontaneous vaginal delivery (SVD). The family history was unremarkable, and the other seven siblings (both males and females) were healthy. Her birth measures (length, weight, occipitofrontal circumference) were within the normal range in reference to her gestational age.

The patient had early healthy motor development, speech delay, and subnormal mentality. At 3-years of age, she was observed to have progressive skeletal changes (Figures 2A,B), with a waddling gait, difficulty to rise-up after sitting down and urine incontinence. History of convulsion or neurological manifestations were not reported.

During the clinical examination, she had good eye contact with no hyperactivity or anxiety. She could understand and obey simple commands and could pronounce a few unclear words. She could walk under assistance. On assessment at the age of 12-years and 7 months, she had short stature $(123 \mathrm{~cm},-4.4 \mathrm{SD})$, lower 


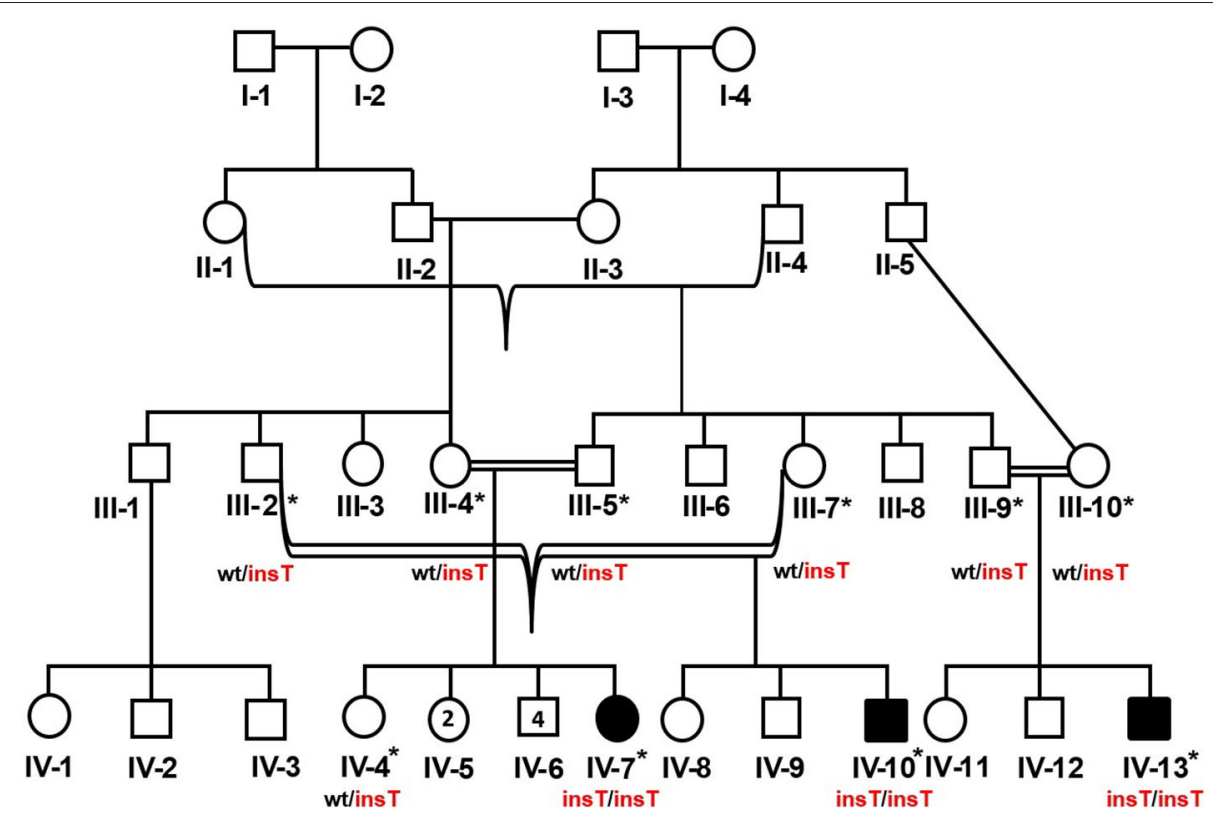

FIGURE 1 | Pedigree of the DMC family in which homozygous insertion variant (c.95_96insT, p.W33Lfs $\left.{ }^{*} 14\right)$ segregates. Available samples are labeled with asterisks, and double lines show consanguineous unions. The red-colored genotype represents the disease allele. The numbers in the symbols show the unaffected male and female siblings of IV-7.

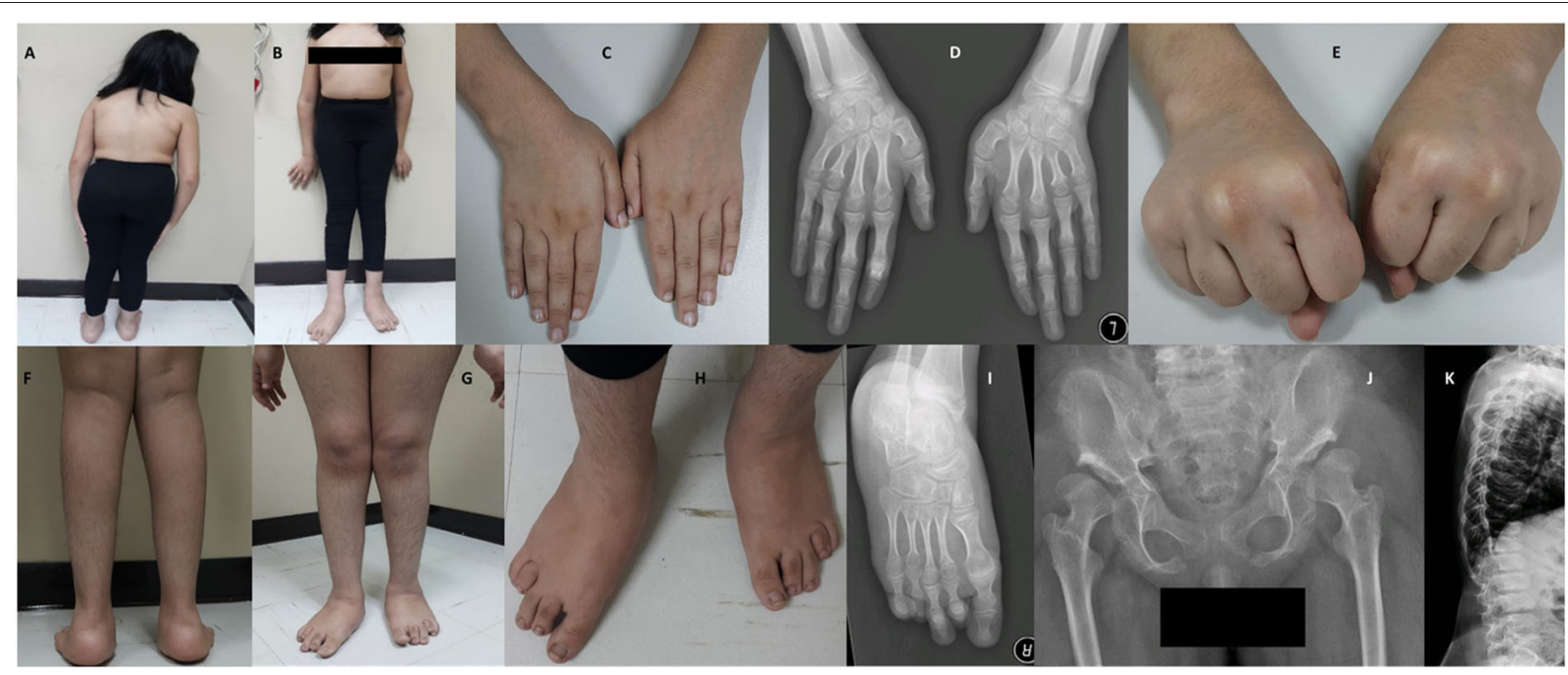

FIGURE 2 | Photos and X-rays of Case-I (IV-7) are showing short trunk, coxavara, mild genu valgum (A,B) short first, fourth and fifth fingers and metacarpals (C,D) and absent fourth and fifth knuckles during fist formation (E), prominent heels, pes planus, brachydactyly of toes more prominent 4th and 5th with short metatarsals (F-I), A-P hips X-rays shows bilateral shallow saucerized acetabulum with abnormally flat and enlarged femoral head and narrow femoral neck, lace-like small iliac wings and bilateral coxa valga (J). X-rays of spines show double hump appearance with antero-inferior beaking of the vertebral bodies (K).

segment (LS) $67 \mathrm{~cm}$, upper to lower segment ratio (US/LS) 0.83 (standard $\sim 0.9$ ), skull circumference was on the 26 th percentile $(53.5 \mathrm{~cm},-0.63 \mathrm{SD})$ and weight was on a fifth percentile $(32 \mathrm{~kg})$. She had low hairline, broad chest, broad elbow, and broad wrist joints (Figures 2B-E). Short and broad hands with brachydactyly of first, fourth, and fifth fingers, with absent fourth and fifth knuckles during fist formation (Figures 2C-E). The lower limbs showed bilateral coxa valga, pes plans, prominent heels, bilateral brachydactyly of fourth and fifth toes (more in fourth), bilateral camptodactyly of second and third toes, and bilateral wide sandal gap (Figures 2F-I). The radiological findings coincided with the clinical reports; the radiological analysis revealed the abnormal development of hip joints and bones (Figure 2J). $\mathrm{X}$-rays of spines revealed a double hump appearance with 
antero-inferior beaking of the vertebral bodies (Figure $\mathbf{2 K}$ ). The patient had an intellectual disability (IQ $=60)$ and generalized familial hypertrichosis. No corneal clouding, hearing deficit, or involvement of other systems were identified.

\section{Case-II (IV-10)}

A 13-year-old male patient (IV-10) was born to healthy double first cousin parents following an uncomplicated pregnancy and vaginal delivery. At the time of his conception, father (III-2) and the mother (III-7) were 56- and 40-years of age. There was a history of two neonatal deaths (a male and a female) with unknown cause, two unaffected siblings (IV-8, IV-9), and two affected cousins (IV-7, IV-13). The patient (IV-10) had an early global developmental delay, with progressive skeletal changes and abnormal gait, observed at the age of two.

He was short $115 \mathrm{~cm}(-5.6 \mathrm{SD})$ and microcephalic, with a head circumference of $48 \mathrm{~cm}(-4.16 \mathrm{SD})$. His weight was on the 10 th percentile $(36 \mathrm{~kg}$ ), U/S 0.77 (normal $\sim 0.9$ ), coarse facial features, low hairline, and prominent lips. He had a short trunk, short chest, broad elbow, and wrist joints, and asymmetric genu valgum more appreciated on the right side (Figures 3A-C). He showed short, broad hands and feet with brachydactyly of all fingers and toes equally, unlike his cousin (case-I, IV7), pes planus, prominent heels, and generalized hypertrichosis (Figures 3C,D). Radiological analysis of the hip joint revealed shallow acetabulum with abnormally flat and enlarged femoral head and narrow femoral neck, lace-like small iliac wings, and bilateral coxa valga (Figure 3E). Intellectual disability with an IQ $=41$ was recorded. Abnormalities of other body organs, visual and hearing impairment were not observed.

\section{Case-III (IV-13)}

A 4-year-old male patient (IV-13), third offspring of the first cousin parents (III-9, III-10), belonging to the same family tree, had major complaints of not gaining height, delay in developmental milestones, and intellectual disability. On physical examination, mild short trunk, broad chest, pes plans, and microcephaly (Figure 3F) were observed. He had a height of $94 \mathrm{~cm}(-2.25 \mathrm{SD})$, lower segment $49 \mathrm{~cm}$, U/L segment 0.92 (normal $\sim 1.2$ ), weight $11 \mathrm{~kg}(<3 \mathrm{rd}$ centile), and skull circumference $43 \mathrm{~cm} /-4.86 \mathrm{SD}$.

\section{Investigations}

Karyotyping, erythrocyte sedimentation rate (ESR), C-reactive protein, ANA (antinuclear antibodies), complete blood count, coagulation profile, GAG (glycosaminoglycans) in the urine, urea and electrolytes, thyroid function tests, parathyroid hormone, serum calcium, phosphorus, and alkaline phosphatase, liver enzymes were all within normal ranges as compared to the reference values, in the three affected members (IV-7, IV10, IV-13). The echocardiogram and abdominal ultrasound were unremarkable.

\section{Screening for a Pathogenic Variant}

DNAs of all three affected members (IV-7, IV-10, IV-13) were subjected to PCR amplification of all 16 coding exons of DYM. DNA sequencing revealed a novel insertion variant (c.95_96insT,
p.W33Lfs*14) in the first coding exon (Figure 4). This variant was present in a heterozygous state in unaffected family members (III-2, III-4, III-5, III-7, III-9, III-10, IV-4), confirming its segregation within the family. The variant was not present in 96 healthy controls of Pakistani population. To date, MAF of this variant is not cited in gnomAD. MutationTaster and PROVEAN (pathogenicity score: -8.10 ) have predicted this novel alteration (c.95_96insT) as a frameshift variant causing a deleterious effect on protein structure. Due to the unavailability of the crystal structure of DYM and reliable templates via PSI-BLAST (https:// www.ebi.ac.uk/Tools/sss/psiblast/) search, the structural studies to investigate the impact of the pathogenic variant were not carried out.

\section{DISCUSSION}

Our study includes three related DMC patients (IV-7, IV-10, IV13). DMC includes various phenotypes like short stature, short trunk, and intellectual disability. Additional symptoms of DMC are microcephaly, coarse facial feature, rhizomelia, and bone deformity $(5,11)$.

During the clinical surveys of our patients, including body examination, and radiological investigations, a clinical feature of rhizomelic shortening was not established in all cases, and case-I (IV-7) did not manifest microcephaly. These are the prominent features of DMC patients, as described in several previous studies $(4,5,10,13)$. The manifestation of mild to severe intellectual disability in all three affected members and microcephaly in male patients, helped us to categorize them as DMC patients instead of SMC patients. The absence of deafness and corneal opacity excluded the chances of Morquio's disease (A and B type), which is caused by the deficiency of $\mathrm{N}$-acetylgalactosamine-6-sulfatase and beta-galactosidase. The excretion of these enzymes in urine was within the normal range in our patients.

Coarse facial features were prominent in case-II and case-III (IV-10, IV-13) in comparison to the female patient (case-I, IV-7). Aglan et al. (4) have reported similar findings in their patients; in which coarse facial feature was more severe in their male cases than the affected females. On the other hand, the female index (case-I) showed significant bilateral shortening of first, fourth, and fifth fingers, and fourth and fifth toes. To the best of our knowledge, this is a distinct clinical feature not described in already reported DMC cases.

DNA sequencing has identified a novel homozygous frameshift variant (c.95_96insT, p.W33Lfs*14) in DYM in affected members. DYM variants have already been described in the literature as the leading cause of the DMC phenotype.

DYM has been mapped at chromosome 18q21.1, encoding a 669 amino acids protein. DYMECLIN (DYM) plays an important role in the regulation of the organization of the Golgi apparatus and morphogenesis of bones (14). To date, HGMD Professional 2018.4 (http://www.hgmd.cf.ac.uk/ac/gene. php?gene $=$ DYM) has a registry of forty-two pathogenic variants [missense/non-sense (17 variants), splicing substitutions (11 variants), deletions (10 variants), and insertions/duplications (4 variants)] in DYM, causing DMC, SMC1, intellectual disability, 


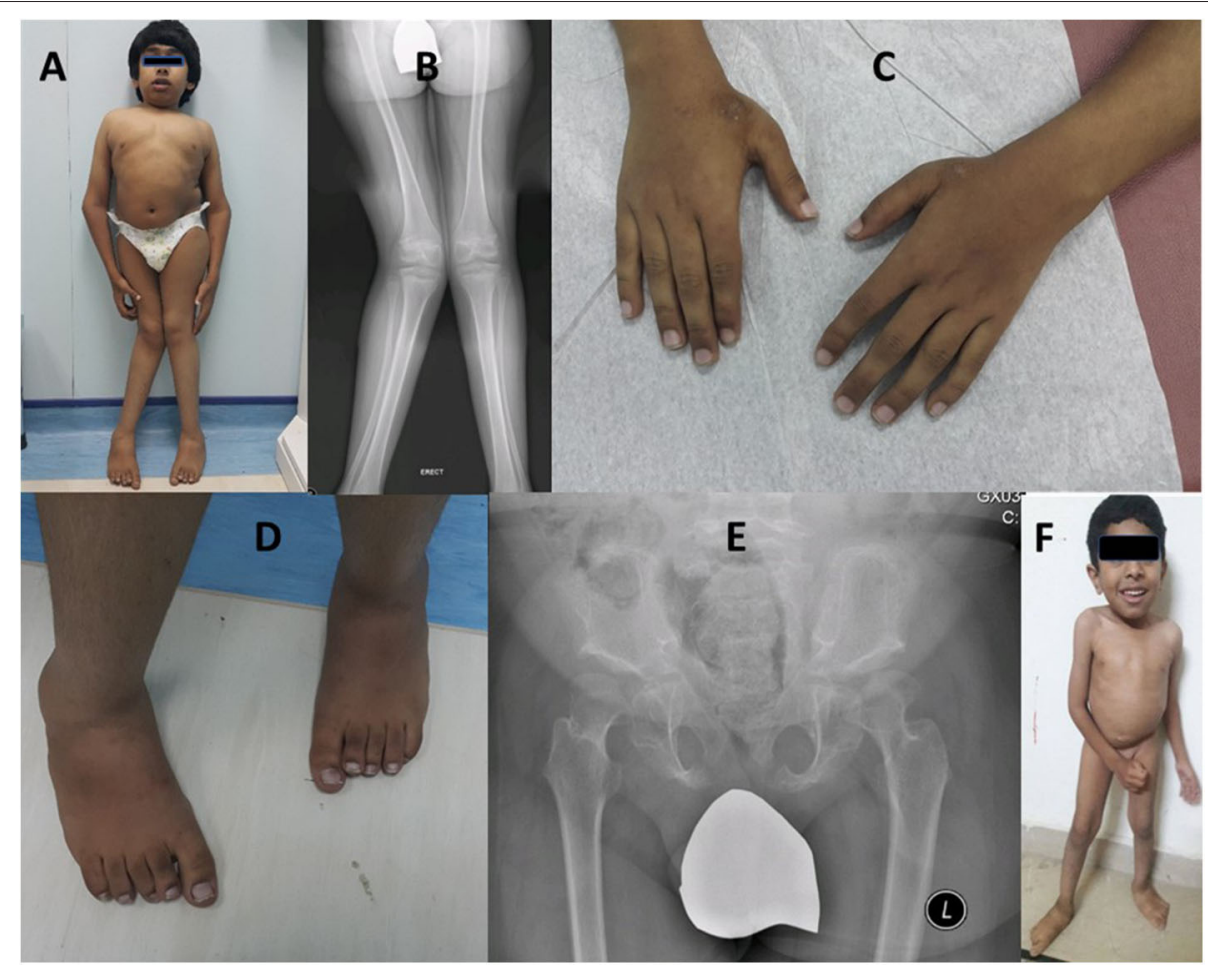

FIGURE 3 | Case-II (IV-10) presenting short trunk, genu valgum more appreciated on the right side (A,B), brachydactyly of fingers (C), short toes with right second toe overriding the first (C,D). Radiological diagram of hip shows shallow acetabulum with abnormally flat and enlarged femoral head and narrow femoral neck, lace-like small iliac wings, and bilateral coxa valga (B,E). Case-lll is a 4-year-old boy (IV-13) showing short trunk, broad chest, and pes planus (F).

A. AATCTATCTCTGAGAATGACC G T T T T G GAATCAGCT T C T T CATT T TCT T TCC T G CACCAACTAGC

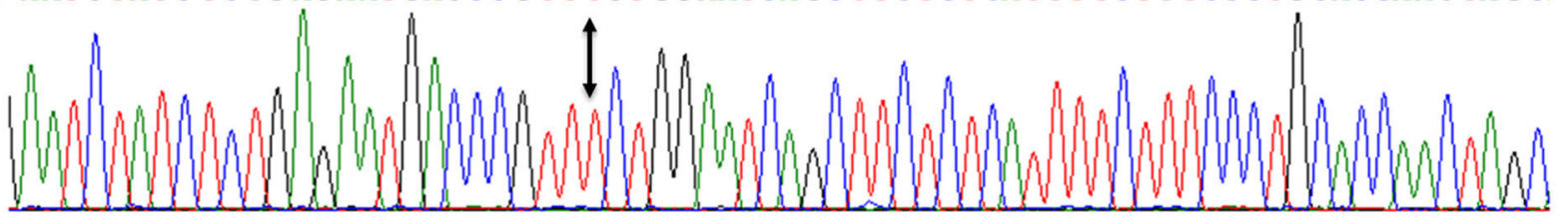

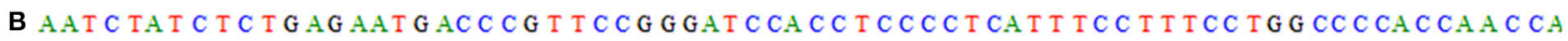

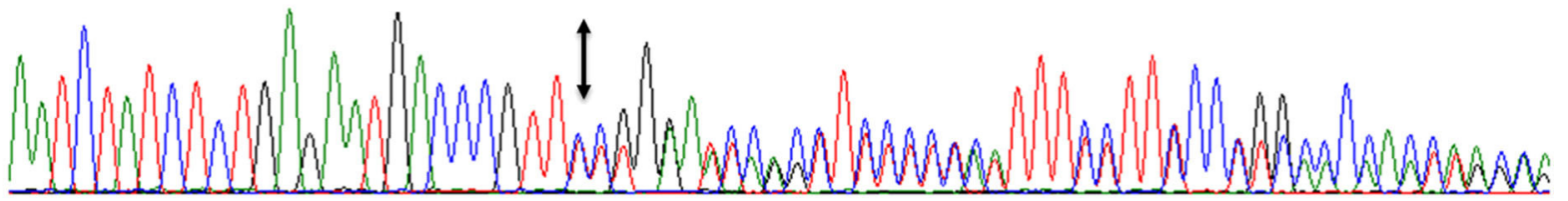

C. AAT C TAT C T C T GAGAAT GAC C C G T T T G GAATCA G C T C T C T CAT T T T T T TCCTGCACCAACTAG CA1

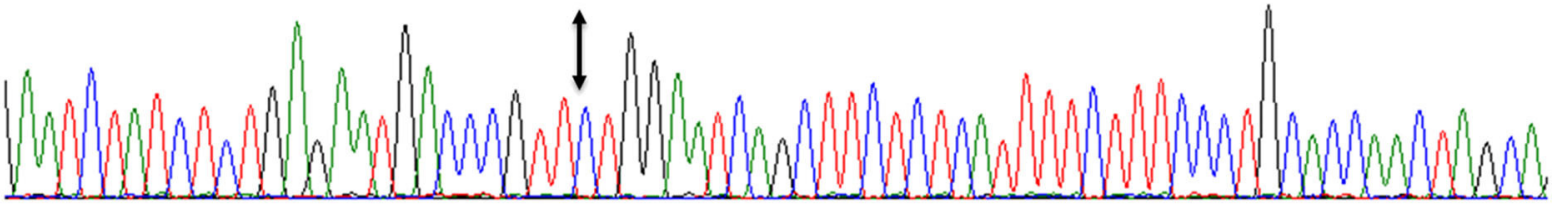

FIGURE 4 | Nucleotide sequence of first coding exon of DYM showing homozygous frameshift variant (c.95_96insT, p.W33Lfs¹4) in an affected member (IV-7) (A), while (B) is showing the DNA sequence of a heterozygous carrier (III-4). (C) is showing a DNA sequence of a control individual. Arrows between the sequences are showing the position of the genetic alteration. The above nucleotide sequences are obtained from same forward primer (DYM-F: $5^{\prime}$-ggtagacaaagcatgatgactttgaac- $3^{\prime}$, melting temperature $64.2^{\circ} \mathrm{C}$ ), used for the Sanger sequencing reaction. 
and other dysmorphic phenotypes. Only twenty-seven DYM variants have been reported to cause DMC (HGMD Professional 2018.4) in patients from various countries around the globe, like Lebanon, Morocco, Tunisia, Pakistan, and India (3, 9).

DYM protein is a non-anchored Golgi protein that shuttles freely from one cellular compartment to another $(7,8,14)$. In a study conducted by Denais et al. (14), it has been described that DYM may have an association with some structural Golgi proteins called soluble NSF [N-ethylmaleimide (NEM)-sensitive fusion protein] attachment protein receptors (SNAREs) and Golgi reassembly stacking proteins (GRASPs) (15-17). These structural proteins play their role in the formation of Golgi ribbons and in developing interactions between these ribbons (14). In the same study, it is illustrated that genetic alterations in genes, encoding regulatory protein for secretion and maintenance of Golgi structure, may affect the development of skeletal muscles (14). In recently published review articles, Passemard et al. (18) and Rasika et al. (19)have classified DMC syndrome as a part of a newly described group of Golgipathies, characterized by skeletal and intellectual defects and caused by disease-causing variants in several Golgi proteins, including DYM. Electron microscopic analysis of chondrocytes and cutaneous cells of affected individuals revealed the dilated cisternae of the rough endoplasmic reticulum (9, 20 ), which may suggest DMC as a storage disease (2). This enlargement of rough endoplasmic reticulum may lead to inadequate extracellular matrix formation, which can be a leading cause of impaired bone morphogenesis in DMC and SMC patients (14).

In the current study, we have identified the fifth homozygous insertion variant (c.95_96insT, p.W33Lfs*14) in DYM in a consanguineous family of Pakistani origin. According to MutationTaster, this frameshift variant may lead to NMD, likely to result in the complete absence of DYM protein. NMD is a conserved mammalian surveillance system which destroys the imperfect messages, generated by premature stop codons (21). Dimitrov et al. (7), have explained that the pathogenic variations in DYM with premature stop codons (PTC), either in the form of non-sense, splice site, deletions, or insertions, may lead to mislocalization and/or degradation of DYM protein in DMC patients.

\section{REFERENCES}

1. Beighton P. Dyggve-Melchior-Clausen syndrome. J Med Genet. (1990) 27:512. doi: 10.1136/jmg.27.8.512

2. Paupe V, Gilbert T, Le Merrer M, Munnich A, Cormier-Daire V, El Ghouzzi V. Recent advances in Dyggve-Melchior-Clausen syndrome. Mol Genet. (2004) 83:51-9. doi: 10.1016/j.ymgme.2004. 08.012

3. Kinning E, Tufarelli C, Winship W, Aldred M, Trembath RC. Genomic duplication in Dyggve Melchior Clausen syndrome, a novel mutation mechanism in an autosomal recessive disorder. J Med Genet. (2005) 42:e70. doi: 10.1136/jmg.2005.033829

4. Aglan MS, Temtamy SA, Fateen E, Ashour AM, Eldeeb K, Hosny GA. Dyggve-Melchior-Clausen syndrome: clinical, genetic, and radiological study
The present study increases the mutation spectrum of DYM and confirms the notion that the deleterious variants causing PTC are the leading cause of human DMC.

\section{DATA AVAILABILITY STATEMENT}

The datasets generated for this study can be found in the ClinVar, SUB6990858, https://submit.ncbi.nlm.nih.gov/subs/variation_ clinvar/SUB6990858/ (accession number SCV001161768).

\section{ETHICS STATEMENT}

This study was approved, under a reference number $24 / 14$, by the medical ethics and research committee of King Abdulaziz University, Jeddah, Saudi Arabia. Written informed consent to participate in this study was provided by the participants' legal guardian/next of kin. Written informed consent was obtained from the individual(s), and minor(s)' legal guardian/next of kin, for the publication of any potentially identifiable images or data included in this article.

\section{AUTHOR CONTRIBUTIONS}

NG, KA, TS, JA-A, and LA have enrolled the patients and contributed to the radiological analysis, clinical diagnoses, and report writing. NW contributed to Sanger Sequencing. NG and AP have written the initial draft of the manuscript. NW, AA, and NG have critically reviewed and finalized the manuscript. All authors read and approved the final draft.

\section{FUNDING}

Naveed Wasif was financially supported by the George Forster Fellowship (2015-2018) of the Alexander von Humboldt Foundation, Bonn, Germany.

\section{ACKNOWLEDGMENTS}

We are thankful to the participants for their generous contribution to this study. We are grateful to the Alexander von Humboldt Foundation, Bonn, Germany, for their financial support.

of 15 Egyptian patients from nine unrelated families. J Pediatr Orthop. (2009) 3:451-8. doi: 10.1007/s11832-009-0211-8

5. Gupta V, Kohli A, Dewan VJ. Dyggve-Melchior-Clausen syndrome. Indian Pediatr. (2010) 47:973-5. doi: 10.1007/s13312-010-0151-x

6. Dyggve H, Melchior J, Clausen J. Morquio-Ullrich's disease: an inborn error of metabolism? Arch Dis Child. (1962) 37:525. doi: 10.1136/adc.37.195.525

7. Dimitrov A, Paupe V, Gueudry C, Sibarita JB, Raposo G, Vielemeyer O, et al. The gene responsible for Dyggve-Melchior-Clausen syndrome encodes a novel peripheral membrane protein dynamically associated with the Golgi apparatus. Hum Mol Genet. (2009) 18:440-53. doi: 10.1093/hmg/ddn371

8. Osipovich AB, Jennings JL, Lin Q, Link AJ, Ruley HE. DyggveMelchior-Clausen syndrome: chondrodysplasia resulting from defects in intracellular vesicle traffic. Proc Natl Acad Sci USA. (2008) 105:16171-6. doi: $10.1073 /$ pnas. 0804259105 
9. El Ghouzzi V, Dagoneau N, Kinning E, Thauvin-Robinet C, Chemaitilly W, Prost-Squarcioni C, et al. Mutations in a novel gene Dymeclin (FLJ20071) are responsible for Dyggve-Melchior-Clausen syndrome. Hum Mol Genet. (2003) 12:357-64. doi: 10.1093/hmg/ddg029

10. Cohn DH, Ehtesham N, Krakow D, Unger S, Shanske A, Reinker K, et al. Mental retardation and abnormal skeletal development (Dyggve-MelchiorClausen dysplasia) due to mutations in a novel, evolutionarily conserved gene. Am J Med Genet. (2003) 72:419-28. doi: 10.1086/346176

11. Dupuis N, Lebon S, Kumar M, Drunat S, Graul-Neumann LM, Gressens P, et al. A novel RAB33B mutation in Smith-McCort dysplasia. Hum Mutat. (2013) 34:283-6. doi: 10.1002/humu.22235

12. Alshammari MJ, Al-Otaibi L, Alkuraya FS. Mutation in RAB33B, which encodes a regulator of retrograde Golgi transport, defines a second Dyggve-Melchior-Clausen locus. J Med Genet. (2012) 49:455-61. doi: 10.1136/jmedgenet-2011-100666

13. Seven M, Koparir E, Gezdirici A, Aydin H, Skladny H, Fenercioglu E, et al. A novel frameshift mutation and infrequent clinical findings in two cases with Dyggve-Melchior-Clausen syndrome. Clin Dysmorphol. (2014) 23:1-7. doi: 10.1097/MCD.0000000000000020

14. Denais C, Dent CL, Southgate L, Hoyle J, Dafou D, Trembath RC, et al. Dymeclin, the gene underlying Dyggve-Melchior-Clausen syndrome, encodes a protein integral to extracellular matrix and Golgi organization and is associated with protein secretion pathways critical in bone development. Hum Mutat. (2011) 32:231-9. doi: 10.1002/humu.21413

15. Shorter J, Warren G. A role for the vesicle tethering protein, p115, in the postmitotic stacking of reassembling Golgi cisternae in a cell-free system. J Cell Biol. (1999) 146:57-70. doi: 10.1083/jcb.146.999.57

16. Shorter J, Warren G. Golgi architecture and inheritance. Annu Rev Cell. (2002) 18:379-420. doi: 10.1146/annurev.cellbio.18.030602.133733
17. Wang Y, Seemann J, Pypaert M, Shorter J, Warren G. A direct role for GRASP65 as a mitotically regulated Golgi stacking factor. EMBO J. (2003) 22:3279-90. doi: 10.1093/emboj/cdg317

18. Passemard S, Perez F, Colin-Lemesre E, Rasika S, Gressens P, El Ghouzzi V. Golgi trafficking defects in postnatal microcephaly: the evidence for "Golgipathies". Prog Neurobiol. (2017) 153:46-63. doi: 10.1016/j.pneurobio.2017.03.007

19. Rasika S, Passemard S, Verloes A, Gressens P, El Ghouzzi V. Golgipathies in neurodevelopment: a new view of old defects. Dev Neurosci. (2018) 40:396416. doi: 10.1159/000497035

20. Nakamura K, Kurokawa T, Nagano A, Nakamura S, Taniguchi K, Hamazaki M. Dyggve-Melchior-Clausen syndrome without mental retardation (SmithMcCort dysplasia): morphological findings in the growth plate of the iliac crest. Am J Med Genet. (1997) 72:11-7.3.

21. Hentze MW, Kulozik AE. A perfect message: RNA surveillance and nonsensemediated decay. Cell. (1999) 96:307-10. doi: 10.1016/S0092-8674(00)80542-5

Conflict of Interest: The authors declare that the research was conducted in the absence of any commercial or financial relationships that could be construed as a potential conflict of interest.

Copyright (C) 2020 Gaboon, Parveen, Ahmad, Shuaib, Al-Aama, Abdelwehab, Arif and Wasif. This is an open-access article distributed under the terms of the Creative Commons Attribution License (CC BY). The use, distribution or reproduction in other forums is permitted, provided the original author(s) and the copyright owner(s) are credited and that the original publication in this journal is cited, in accordance with accepted academic practice. No use, distribution or reproduction is permitted which does not comply with these terms. 